\title{
Quadrijunctions-stunted grain growth in duplex microstructure: A multiphase-field analysis
}

\author{
Ramanathan Perumal ${ }^{\mathrm{a}, \mathrm{b}, 1}, \mathrm{P}$ G Kubendran Amos $^{\mathrm{a}, 1, *}$, Michael Selzer ${ }^{\mathrm{a}, \mathrm{b}}$, Britta Nestler ${ }^{\mathrm{a}, \mathrm{b}}$ \\ ${ }^{a}$ Institute of Applied Materials (IAM-CMS), Karlsruhe Institute of Technology (KIT), \\ Strasse am Forum 7, 76131 Karlsruhe, Germany \\ ${ }^{b}$ Institute of Digital Materials Science (IDM), Karlsruhe University of Applied Sciences, \\ Moltkestr. 30, 76133 Karlsruhe, Germany
}

\begin{abstract}
Influence of energetically-preferred quadrijunctions on the kinetics of grain growth in textured two-dimensional duplex-microstructure is investigated in this work. A multiphase-field approach, wherein individual grains are associated with the constituent phases of the microstructure through appropriate initialisation, is adopted for the present study. Different forms of anisotropy, phase-dependent and -independent, established by appropriately varying grain boundary energies are considered. While being consistent with the analytical predictions, and existing studies, the present investigation unravels that, irrespective of the nature of anisotropy, the kinetics of the microstructural evolution in duplex polycrystalline system linearly decreases with increase in the density of quadrijunctions.
\end{abstract}

Keywords: Quadrijunctions, quadruple-junctions, stunted grain growth, concurrent grain growth and coarsening, duplex microstructure

Polycrystalline microstructures are generally characterised by grain boundaries, the interface separating two grains, and by triple junctions, wherein three grain boundaries meet. While the misorientation between the adjoining grains dictates the corresponding grain boundary energy [1], the local configuration of the triple junction, particularly, the angle between grain boundaries is governed by their energies [2]. The ability of the system to reduce its interfacial energy-density (per unit volume), under appropriate thermodynamical condition, results in

\footnotetext{
*P G Kubendran Amos

Email address: prince.amos@kit.edu (P G Kubendran Amos)

${ }^{1}$ The authors contributed equally.
} 
grain growth. Evolution of a grain, locally, is influenced by numerous geometrical and topological factors including size and face-class [3, 4]. However, differences in grain boundary energy effect both the overall evolution of the microstructure, often leading to an abnormal growth [5], and the local arrangement of grains at the triple junctions [6]. In order to introduce anisotropy in grain boundary energies, theoretical treatments broadly consider several 'classes' of grains, and assign different energies to the boundaries separating grains of the similar and dissimilar classes [7, 8]. Although such approach is adopted for the ease of incorporating different grain boundary energies, multiphase polycrystalline systems with chemically-distinct grains inherently render a suitable setup for incorporating anisotropy in grain boundary energies [9].

As opposed to a conventional polycrystalline system, wherein grains are chemically identical, each grain is exclusively associated with one of the constituent phases in a multiphase microstructure [10, 11]. Therefore, grain growth in a multiphase system is implicitly constrained to preserve the characteristic phase-fractions. Moreover, while the kinetics of evolution is dictated by the rate of interface migration in regular polycrystalline systems, the diffusion of mass governs the grain growth rate in multiphase microstructures [12, 13]. Despite these complexities, multiphase polycrystalline system offers an ideal setup for understanding the influence of anisotropy in grain boundary energies on energy-minimising evolution. Particularly, in a textured microstructure, wherein the energies of boundary separating grains of a given phase are almost equal, the anisotropy is primarily associated with the boundaries of chemically-distinct grains [14].

Effect of anisotropy in grain boundary energies is distinct in multiphase systems when compared to regular polycrystalline microstructures. The constraint pertaining to the phase-fraction averts the abnormal growth of energetically-favoured grains. However, the differences in grain boundary energies dictate the distribution of grains, and correspondingly, influence the overall microstructural evolution. One interesting effect of this anisotropy is the formation of the stable quadruple-junctions or quadrijunctions, where four grains interact [15]. The stability of quadrijunctions, its introduction in multiphase polycrystalline system, and its effect of mechanism of evolution have been analytically and numerically analysed in microstructures with conserved [16, 12] and non-conserved phase-fractions [17, 18, 19]. Despite the expanse of these 
investigations, the influence of quadrijunctions on the kinetics of microstructural evolution in multiphase systems, though experimentally observed [20], is yet to be reported. Therefore, in this work, multiphase-field approach is employed to investigate the effect of grain boundary anisotropy, through the formation of stable quadrijunctions, on the transformation rate of textured two-dimensional duplex microstructure [21, 22].

In addition to phase transformation including solid-state evolution [23, 24, 25], phase-field treatment has been involved in analysing energy-minimising curvature-driven morphological transformations [26, 27]. An efficient alternate of preserving volume through a bulk contribution [28, 29], instead of treating phase-field as conserved variable [30], is increasingly adopted to understand volume-preserved microstructural changes. Similar approach in a multiphase-field framework, which has asymptotically been proven to recover sharp-interface solutions [31], is employed in the present study [32]. For a comprehensive understanding of the multiphase-field treatment, the readers are directed to Ref [33, 34, 35].

Identical two-dimensional domains with uniform grids of size $\Delta \mathrm{x}=\Delta \mathrm{y}=1.0$ and dimension $2048 \times 2048$ is considered for all microstructures in the current analysis. A polycrystalline setup is established by Voronoi tessellation, which for given size of the domain renders approximately 10000 grains. Individual grains are associated with the constituent phases by assigning an appropriate conserved variable (concentration) and energy-density. Since this study primarily focuses on understanding the effect of anisotropy in grain boundary energy, diffusivity is treated as a constant $(D=1.0)$. Moreover, all duplex microstructures considered in the present work comprise of equal volume-fraction of the phases.

In textured duplex-microstructure, the anisotropy in grain boundary energy can be quantified by two parameters, $E_{\alpha}$ and $E_{\beta}$, where $E_{\alpha}=\frac{\gamma_{\alpha \alpha}}{\gamma_{\alpha \beta}}$ and $E_{\beta}=\frac{\gamma_{\beta \beta}}{\gamma_{\alpha \beta}}[15,13]$. Considering the textured nature of the system, the energies of boundary separating chemically-similar and -dissimilar grains are denoted, without complexities, by $\gamma_{\alpha \alpha}\left(\right.$ or $\left.\gamma_{\beta \beta}\right)$ and $\gamma_{\alpha \beta}$, respectively [14]. The number density of quadrijunctions, $\mathcal{Q}$, formed during the evolution of microstructures with different $E_{\alpha}$ $\left(=E_{\beta}\right)$ is shown in Fig 1 . Duplex microstructures associated with each $E_{\{\alpha, \beta\}}$, after the evolution reaches a self-similar state, is included in the illustration. The anisotropy parameters $E_{\{\alpha, \beta\}}$ are varied by fixing the boundary energy of dissimilar grains, and changing $\gamma_{\alpha \alpha}$ and $\gamma_{\beta \beta}$. 


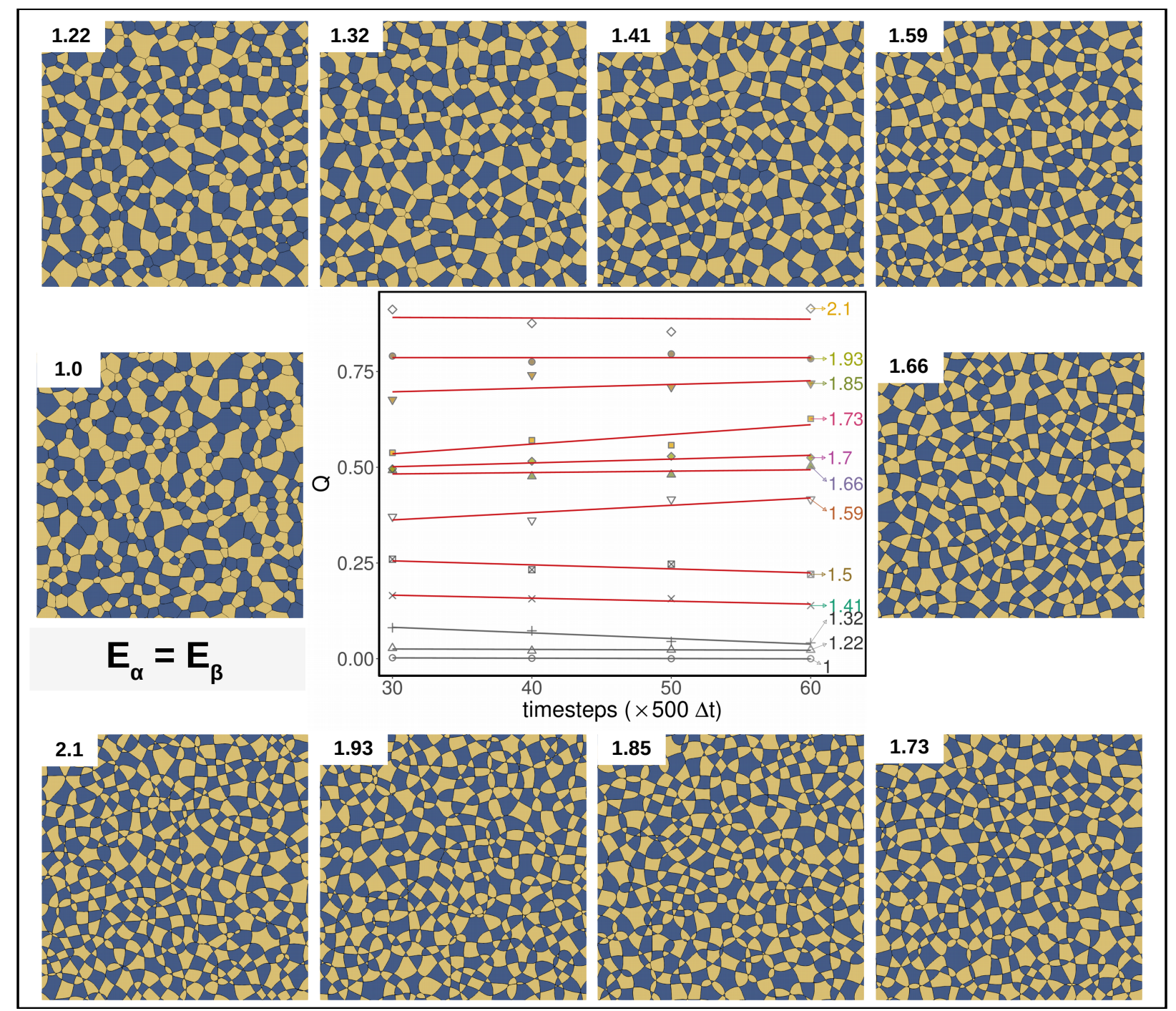

Figure 1: Number fraction quadruple-junction, $\mathcal{Q}$, calculated as the ratio of the number of quadri- and total-junctions, for different anisotropic condition at various non-dimensional timesteps. Microstructures illustrating the distribution of phase-associated grains for varied anisotropy is included. 
In complete agreement with existing studies [19, 16], Fig. 11 shows that quadrijunctions are almost non-existent in isotropic condition characterised by $\gamma_{\alpha \alpha}=\gamma_{\beta \beta}=\gamma_{\alpha \beta}=1$. However, noticeable amount of $\mathcal{Q}$ is formed in system with $E_{\{\alpha, \beta\}}=1.22$ and 1.32, which seemingly contradicts the analytical claim that stable quadrijunctions are formed only when $E_{\{\alpha, \beta\}} \geq \sqrt{2}[15]$. A previous study, wherein quadruple-junctions were observed in polycrystalline system with 70 $E_{\{\alpha, \beta\}}<\sqrt{2}$, attributes the deviation from the criterion to the limitation of the analytical treatment [14]. Although this argument is apparently convincing, it should be noted that experimental observations [20, 36, 37], and theoretical studies [38, 16], unravel the formation of quadrijunctions during disappearance of four face-class grains, irrespective of $E_{\{\alpha, \beta\}}$. Despite their dissociation into two triple points, the rate of the dissociation is influenced by several factors, and often sluggish in multiphase anisotropic systems [39,40]. Therefore, in a given timestep, these 'transitory', and more importantly, unstable quadrijunctions appear as stable. Owing to the lack of a clear distinction between the transitory and stable quadrijunctions, it is inaccurate to claim that all the observed quadrijunctions are stable at any moment of grain growth. Furthermore, based on the progressive decrease in $\mathcal{Q}$ for $E_{\{\alpha, \beta\}}=1.32$ in Fig. 1 it can be suggested that the corresponding quadrijunctions are largely transitory in nature.

Influence of grain-boundary energy anisotropy on the distribution of the phase-associated grains is evident in Fig. 1 . Since grain boundary energies, irrespective of the chemical make-up of grains, are equal in isotropic condition, the distribution is apparently random. However, as the anisotropy is introduced by relatively increasing $\gamma_{\alpha \alpha}$ and $\gamma_{\beta \beta}$ in relation to $\gamma_{\alpha \beta}$, clusters of chemically-similar grains are averted, and boundaries of dissimilar grains are preferred. Moreover, with increase in number-density of quadrijunctions, the dominant face-class shift from 6 to 4 in accordance with Euler-Poincare rule, and 'checker-board' like distribution is established [19, 38].

The kinetics of the microstructural evolution in the duplex microstructures is analysed by monitoring the temporal change in average grain size, $R(t)$. Fig. 2 shows the progressive increase in average grain size of different two-phase microstructures distinguished by the degree of phase-independent grain-boundary energy anisotropy, $E_{\alpha}\left(=E_{\beta}\right)$. Evidently, with increase in the anisotropy $E_{\{\alpha, \beta\}}$, the kinetics of evolution decreases. Despite the lowering of the growth 


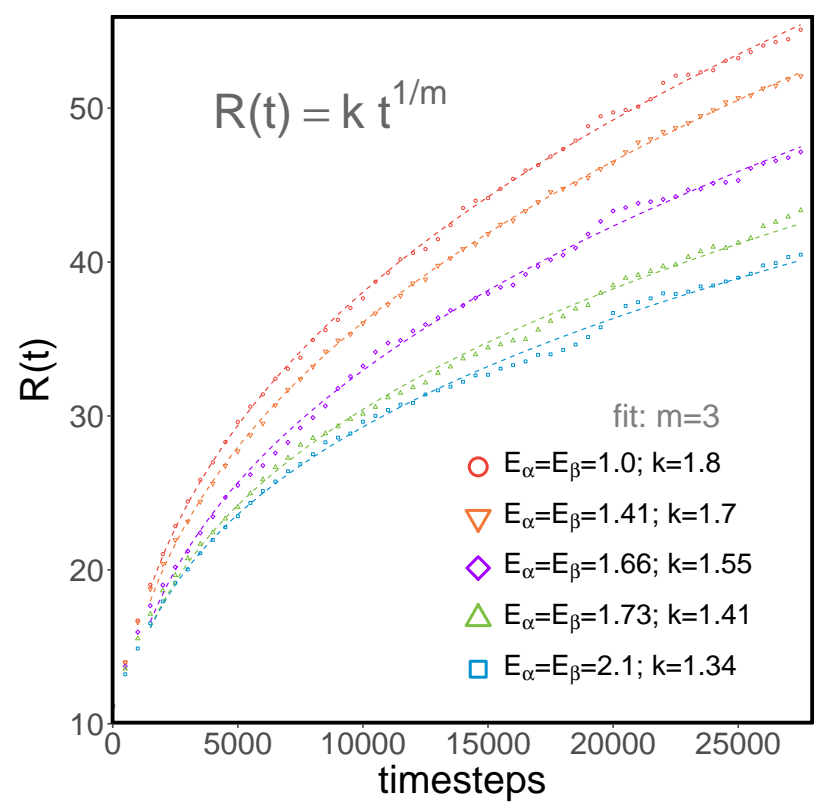

Figure 2: Change in the average grain size of duplex microstructures with time, under various degree of phaseindependent anisotropy in grain boundary energy $\left(E_{\alpha}=E_{\beta}\right)$.

rate, as shown in Fig. 2 all microstructural transformations adhere to the growth law with exponent $m \approx 3$. This similarity in the exponent, irrespective of the differences in the anisotropy, indicates the dominant mechanism of diffusion governed grain-growth [12].

In Fig. 2. except for isotropic systems $\left(E_{\{\alpha, \beta\}}=1.0\right)$, all other duplex microstructures, as indicated in Fig. 1. comprise of considerable amount of quadrijunctions. Moreover, in the polycrystalline setup with $E_{\{\alpha, \beta\}}=2.1$, all junctions are almost exclusively quadruple-points [13]. Initial works investigating the influence of quadrijunctions on grain growth suggested that these junctions are sessile, and consequently, freeze the evolution [19]. However, subsequent theoretical [18, 13] and experimental studies [20, 41] have contradicted this claim, and shown that microstructures continue to evolve despite the predominant presence of quadrijunctions. Consistent with these observations, Fig. 2 shows that the duplex microstructures actively transform despite the presence of significant amount of quadruple-junctions.

Based on Fig. 2, which suggests that the transformation kinetics is influenced by the numberdensity of quadrijunctions, $\mathcal{Q}$, effect of degree of anisotropy on $\mathcal{Q}$ and the rate of microstructural 

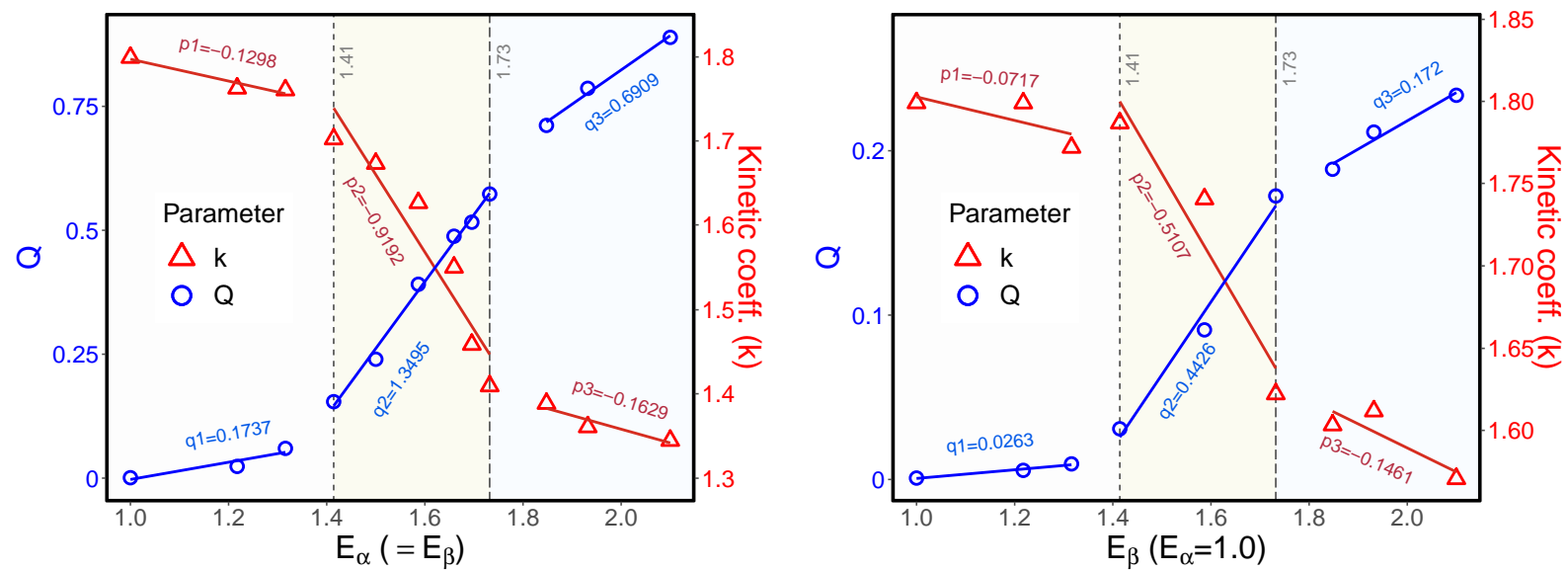

Figure 3: a) Increase in the fraction of quadrijunctions, and consequent decrease in the kinetics of grain growth, with raise in the degree of anisotropy $E_{\alpha}\left(=E_{\beta}\right)$. The slope of the fitting lines are denoted by $p\{1,2,3\}$ and $q\{1,2,3\}$. b) Influence of unequal degree of anisotropy $E_{\alpha} \neq E_{\beta}$ on the quadrijunction number-density and kinetics of microstructural evolution.

evolution is cumulatively plotted in Fig. 3 a. Since grain growth in all duplex microstructures exhibit a constant exponent, the disparity in the kinetics is explicated by the coefficient $k$. The illustration in Fig. $3 \mathrm{a}$ is distinguished into three section in accordance with the analytical relations [15]. First section, characterised by $E_{\{\alpha, \beta\}}<\sqrt{2}$, pertains to the anisotropic condition wherein quadrijunctions are supposedly unstable, and in third section, which is demarcated by $E_{\{\alpha, \beta\}}>\sqrt{3}$, triple junctions of chemically-identical grains are not energetically favoured.

When $E_{\{\alpha, \beta\}}<\sqrt{2}$, although a noticeable density of quadrijunctions is only observed for anisotropy close to $\sqrt{2}$, the kinetics visibly decreases [14]. The reduction in the transformation rate is more pronounced in second section sandwich between $\sqrt{2}>E_{\{\alpha, \beta\}}>\sqrt{3}$, wherein the number-density of quadrijunctions proportionately increase with the anisotropy. Moreover, Fig. 3 a shows that the diminishing of the growth rate beyond the second section, $E_{\{\alpha, \beta\}}>\sqrt{3}$, does not correspond to the considerable increase in quadrijunctions. In other words, when the duplex microstructure consists of a combination of triple- and quadruple-junctions, the transformation kinetics is highly sensitive to $\mathcal{Q}$. While the evolution is predominantly dictated by stable quadrijunctions, the effect of $\mathcal{Q}$ on the growth rate is reduced. This behaviour can primarily be attributed to the varying stability of the junctions under different anisotropic conditions [15, 13]. 


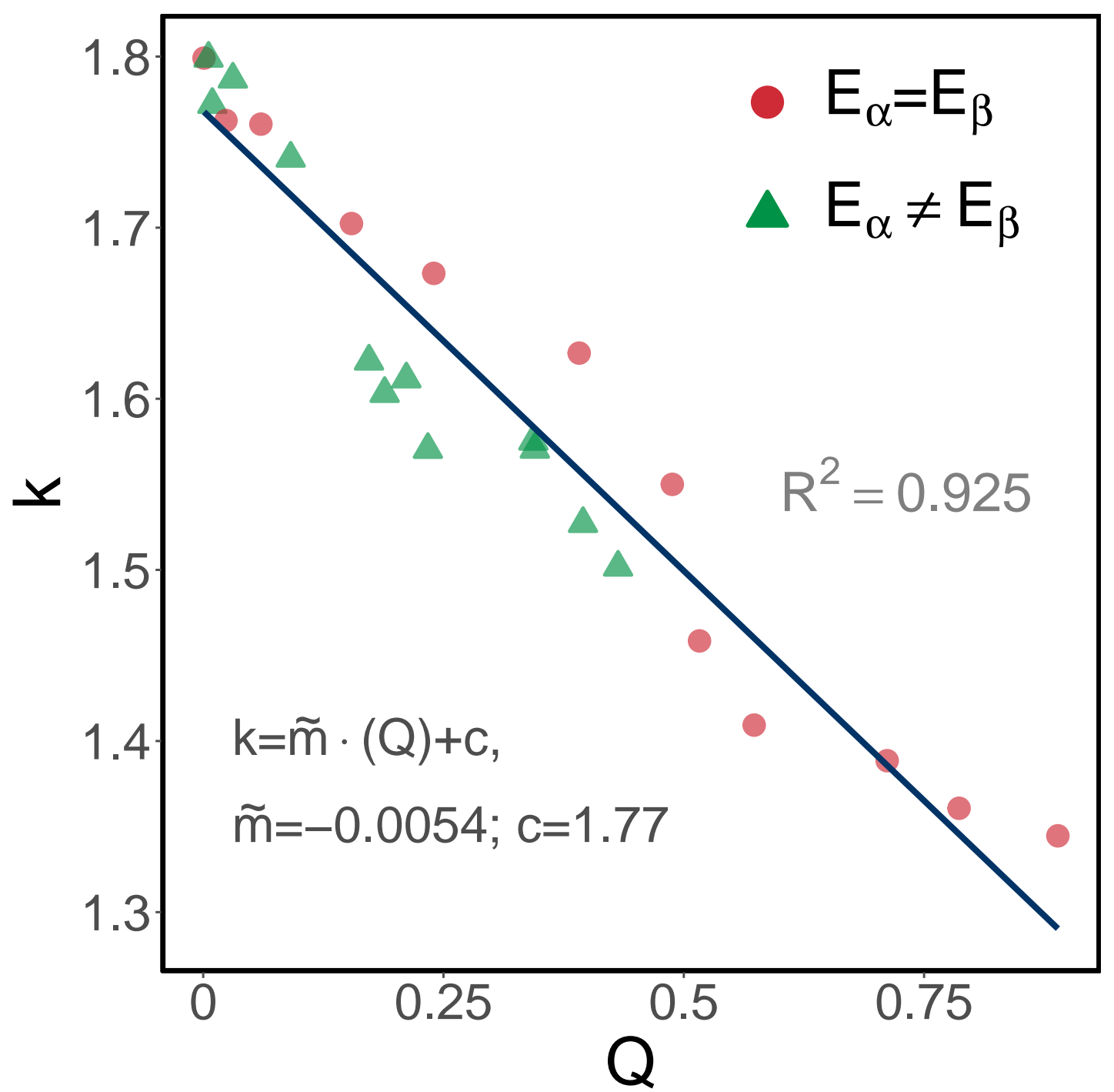

Figure 4: Change in the kinetic coefficient of evolving duplex microstructures with increase in the number-density of quadruple-junctions. 
In order to investigate the effect of phase-dependent anisotropic condition, temporal evolution of duplex microstructures with $E_{\alpha} \neq E_{\beta}$ is a analysed. The inequality in the energy-ratios is achieved by fixing $E_{\alpha}=1.0$, while correspondingly varying $E_{\beta}$. Similar to Fig. 3 a, the change in the transformation rate and number-density of quadruple-junction with increase in unequal energy-ratios $\left(E_{\alpha} \neq E_{\beta}\right)$ is plotted in Fig. $3 \mathrm{~b}$. The overall influence of the unequal energyratios on the evolution of duplex microstructure is comparable to the effect of phase-independent anisotropy $E_{\alpha}\left(=E_{\beta}\right)$. In other words, Fig. 3b unravels three sections with each exhibiting a characteristic change in $\mathcal{Q}$ and kinetic coefficient, $k$. While there is only a marginal change in kinetics and number-density of quadrijunctions in first section, the effect of anisotropy is highly significant in second section. In third section, analogous to Fig. 3 a, the influence of anisotropic grain-boundary energy is rather minimised.

In conclusion, the effect of quadruple-junction on grain growth rate of duplex microstructures is directly examined by plotting its number-density against the kinetic coefficient $k$ in Fig. 4 . The influence of phase-dependent and -independent anisotropic conditions, though distinguished, are collectively illustrated. Fig. 4 shows that, irrespective of the nature of grain-boundary energy anisotropy, $E_{\alpha}=E_{\beta}$ or $E_{\alpha} \neq E_{\beta}$, with increase in the density of quadrijunctions, the kinetics of evolution in duplex microstructure decreases proportionately in a linear fashion.

Attempts are currently being made to extend the present analysis to three-dimensional multiphase microstructures with particular aim to understand the distribution and kinetics of evolution.

\section{Acknowledgments}

PGK Amos and R Perumal respectively thank the financial support of the German Research Foundation (DFG) under the project AN 1245/1 and the BMBF project 'KerSOLife100'. Support of the Helmholtz association through the programme 'Renewable energies RE 35.14 ' is gratefully acknowledged. 


\section{References}

[10] Robert Gunn. Duplex stainless steels: microstructure, properties and applications. Elsevier, 1997.

[16] Danan Fan and Long-Qing Chen. Topological evolution during coupled grain growth and ostwald ripening in volume-conserved 2-d two-phase polycrystals. Acta materialia, 45(10):4145-4154, 1997.

[17] JW Cahn, Elizabeth A Holm, and David J Srolovitz. Modeling microstructural evolution in two-dimensional 
two-phase microstructures. In Materials Science Forum, volume 94, pages 141-158. Trans Tech Publ, 1992.

[18] John W Cahn and Erik S Van Vleck. Quadrijunctions do not stop two-dimensional grain growth. Scripta materialia, 34(6):909-912, 1996.

[19] EA Holm, David J Srolovitz, and JW Cahn. Microstructural evolution in two-dimensional two-phase polycrystals. Acta metallurgica et materialia, 41(4):1119-1136, 1993.

[20] Cong-zhang QIU, LIU Yong, Lan HUANG, Wei ZhANG, LIU Bin, and LU Bin. Effect of fe and mo additions on microstructure and mechanical properties of tial intermetallics. Transactions of Nonferrous Metals Society of China, 22(3):521-527, 2012.

[21] Ramanathan Perumal, PG Kubendran Amos, Michael Selzer, and Britta Nestler. Phase-field study on the formation of first-neighbour topological clusters during the isotropic grain growth. Computational Materials Science, 140:209-223, 2017.

[22] Ramanathan Perumal, PG Kubendran Amos, Michael Selzer, and Britta Nestler. Phase-field study of the transient phenomena induced by 'abnormally'large grains during 2-dimensional isotropic grain growth. Computational Materials Science, 147:227-237, 2018.

[23] LT Mushongera, PG Kubendran Amos, B Nestler, and K Ankit. Phase-field simulations of pearlitic divergence in fe-c-mn steels. Acta Materialia, 150:78-87, 2018.

[24] PG Kubendran Amos, Ephraim Schoof, Daniel Schneider, and Britta Nestler. Chemo-elastic phase-field simulation of the cooperative growth of mutually-accommodating widmanstätten plates. Fournal of Alloys and Compounds, 767:1141-1154, 2018.

[25] PG Kubendran Amos, Ephraim Schoof, Nick Streichan, Daniel Schneider, and Britta Nestler. Phase-field analysis of quenching and partitioning in a polycrystalline fe-c system under constrained-carbon equilibrium condition. Computational Materials Science, 159:281-296, 2019.

[26] PG Kubendran Amos, LT Mushongera, Tobias Mittnacht, and Britta Nestler. Phase-field analysis of volumediffusion controlled shape-instabilities in metallic systems-ii: Finite 3-dimensional rods. Computational Materials Science, 144:374-385, 2018.

[27] PG Kubendran Amos, Ephraim Schoof, Daniel Schneider, and Britta Nestler. On the globularization of the shapes associated with alpha-precipitate of two phase titanium alloys: Insights from phase-field simulations. Acta Materialia, 159:51-64, 2018.

[28] PG Kubendran Amos, Ephraim Schoof, Daniel Schneider, and Britta Nestler. On the volume-diffusion governed termination-migration assisted globularization in two-phase solid-state systems: Insights from phase-field simulations. In Numerical Modelling in Engineering, pages 47-63. Springer, 2018.

[29] PG Kubendran Amos, Avisor Bhattacharya, Britta Nestler, and Kumar Ankit. Mechanisms of pearlite spheroidization: Insights from 3d phase-field simulations. Acta Materialia, 161:400-411, 2018.

[30] Junseok Kim, Seunggyu Lee, and Yongho Choi. A conservative allen-cahn equation with a space-time depen- 
dent lagrange multiplier. International fournal of Engineering Science, 84:11-17, 2014.

[31] Prince Gideon Kubendran Amos. Understanding the volume-diffusion governed shape-instabilities in metallic systems. PhD thesis, Karlsruher Institut für Technologie (KIT), 2019.

[32] Tobias Mittnacht, PG Kubendran Amos, Daniel Schneider, and Britta Nestler. Understanding the influence of neighbours on the spheroidization of finite 3-dimensional rods in a lamellar arrangement: Insights from phase-field simulations. In Numerical Modelling in Engineering, pages 290-299. Springer, 2018.

[33] PG Kubendran Amos, Ramanathan Perumal, Michael Selzer, and Britta Nestler. Multiphase-field modelling of concurrent grain growth and coarsening in complex multicomponent systems. Fournal of Material Science and Technology, Accepted, In-Press.

[34] Ramanathan Perumal, Michael Selzer, and Britta Nestler. Concurrent grain growth and coarsening of twophase microstructures; large scale phase-field study. Computational Materials Science, 159:160-176, 2019.

[35] PG Kubendran Amos, LT Mushongera, and Britta Nestler. Phase-field analysis of volume-diffusion controlled shape-instabilities in metallic systems-i: 2-dimensional plate-like structures. Computational Materials Science, 144:363-373, 2018.

[36] Siddhartha Pathak, Roger D Doherty, Anthony D Rollett, Johann Michler, and Kilian Wasmer. Caught in the act: Grain-switching and quadrijunction formation in annealed aluminum. Scripta Materialia, 69(1):37-40, 2013.

[37] N Noel, H Jamgotchian, and B Billia. In situ and real-time observation of the formation and dynamics of a cellular interface in a succinonitrile- $0.5 \mathrm{wt} \%$ acetone alloy directionally solidified in a cylinder. fournal of crystal growth, 181(1-2):117-132, 1997.

[38] Moneesh Upmanyu, Gregory N Hassold, Andrei Kazaryan, Elizabeth A Holm, Yunzhi Wang, B Patton, and David J Srolovitz. Boundary mobility and energy anisotropy effects on microstructural evolution during grain growth. Interface Science, 10(2-3):201-216, 2002.

[39] K Lee and W Losert. Controlled dynamics of grain boundaries in binary alloys. Acta materialia, 53(12):35033510, 2005.

[40] D Fan, SP Chen, and Long-Qing Chen. Computer simulation of grain growth kinetics with solute drag. Fournal of materials research, 14(3):1113-1123, 1999.

[41] AE Mora, JW Steeds, and JE Butler. Grain boundaries in boron-doped cvd diamond films. Diamond and related materials, 11(3-6):697-702, 2002. 